\title{
Nanoemulgel for Transdermal Delivery of Cyclobenzaprine Hydrochloride: Design, Characterization and In-Vitro Studies
}

\author{
Lakshmana Prabu S*, Sharvanan SP, Aravindan S, Bhuvaneswari A and Manikandan V \\ Department of Pharmaceutical Technology, Anna University, India
}

Submission: May 10, 2017; Published: June 22, 2017

*Corresponding author: Lakshmana Prabu S, Department of Pharmaceutical Technology, Bharathidasan Institute of Technology, Anna University, Tiruchirappalli, India, Tel: +91 9750550965; Email: slaxmanvel@gmail.com

\begin{abstract}
Recently utilizing nanoemulsion as a vehicle for deliver the drug through transdermal drug delivery has been increased. The purpose of the present study was to develop a nanoemulsion formulation of cyclobenzaprine hydrochloride and characterize for transdermal drug delivery. Aqueous titration method was adopted for various oil-in-water nanoemulsion preparations. The prepared nanoemulsions were characterized for its particle size, polydispersity index, zeta potential, TEM, $\mathrm{pH}$, viscosity, drug content, spread ability and its in-vitro release studies. Drug excipient compatibility study results reveals that the excipient used in the nanoemulsion is having compatibility. Based on its invitro release studies formulation F14 showed higher release compared to other formulations. Formulation F14 was converted into Nanoemul gel and characterized. The in-vitro release profile of the optimized formulation was compared with the nanoemul gel. In conclusion, the results of the present investigation suggested that the developed nanoemul gel formulation of cyclobenzaprine hydrochloride can be used as a vehicle for enhancement of bioavailability through transdermal drug delivery.
\end{abstract}

Keywords: Cyclobenzaprine hydrochloride; Nanoemulsion; Nanoemul gel; Release studies

\section{Introduction}

An ideal drug delivery system is that fulfills the objective of spatial placement and temporal delivery resulting maximized therapeutic effect and least toxicity. The pharmaceutical scientists are using a wide range of methods to improve the bioavailability of poorly water-soluble drugs coming out of high throughput screening in drug discovery process. With the progress in time and growth of science and technology, the dosage forms have evolved from simple mixtures and pills into highly sophisticated technology intensive systems, which are known as novel drug delivery systems (NDDS). Different approaches have materialized into various forms of NDDS such as microemulsions, multiple emulsions, liposomes, niosomes, micospheres, pharmacosomes, virosomes, dendrimers, etc., Most often the problems associated with these delivery systems are their stability and predictability in biological systems which reduce their clinical potential, although each one is associated with its own strong points $[1,2]$.

Nowadays Nanotechnology is a rapidly expanding field. Various nano-pharmaceuticals currently being used in the process of development are nanoemulsions, nanosuspensions, nanospheres, nanotubes, nanoshells, nanocapsules, lipid nanoparticles and dendrimers [3]. In recent years, one of the most popular approaches is the incorporation of the active lipophilic component into the inert lipid vehicles [4]. Among these drug delivery systems Nanoemulsions has emerged as a boon of nanotechnology in the form of a novel drug delivery system (NDDS). Nanoemulsions are a group of dispersed particles used for pharmaceuticals, biomedical aids, vehicles and also the most valuable options offered to improve the oral bioavailability of poorly water-soluble drugs [5]. Nanoemulsion is considered to be a thermodynamically or kinetically stable liquid dispersion and transparent that shows great promise in the future pharmaceuticals. The most used type of nanoemulsion in pharmaceutical is the system in which the oil (internal) phase is dispersed in an aqueous (external) phase [6].

In Nanoemulsion the drug is loaded into the inner phase of these systems and delivered by lymphatic route, bypassing the enzymes in the gastrointestinal tract (GIT) and reducing the pre systemic clearance and hepatic first pass metabolism. Nanoemulsions have a higher solubilization capacity, better thermodynamic stability, long self-life, rapid onset of action, the nanosized droplets leading to enormous interfacial areas of the drugs associated with nanoemulsions would influence solubilization behavior, transport properties as well as 


\section{Novel Approaches in Drug Designing \& Development}

absorption across the mucosa are an important promising factor to achieve sustained and optimum targeted drug delivery $[7,8]$. Nanoemulsions have been reported to improve the plasma concentration profiles and reduced inter subject bioavailability of drugs more reproducible [9-11].

Cyclobenzaprine hydrochloride is a skeletal muscle relaxant used in the treatment of skeletal muscle relaxant. It is extensively metabolized and excreted primarily as glucronides via kidney and has slow elimination half life of $18 \mathrm{~h}$ and an oral bioavailability of $33 \%$ to $55 \%$. The daily dose ranges from $5 \mathrm{mg}$ to $10 \mathrm{mg}$. It is commercially available as oral tablets. In the present study, to overcome the obstacles in oral drug delivery and to enhance the bioavailability of the cyclobenzaprine, we have developed a novel 0/W type nanoemulsion based gel formulation for the transdermal delivery of cyclobenzaprine hydrochloride without the use of additional permeation enhancers for better applicability, to enhance the local therapeutic potential through the skin.

\section{Materials and Methods}

\section{Drugs and chemicals}

Cyclobenzaprine hydrochloride was obtained as gift samples from Hetero Pharmaceuticals Pvt Ltd., Hyderabad, India; tween 80 , span 80, polyethylene glycol, propylene glycol, carbopol 940, HPMC K15 and isopropyl myristate were procured from Loba Chemie Pvt., Ltd., Mumbai, India; methanol, ethanol, potassium dihydrogen phosphate, sodium hydroxide, hydrochloric acid, triethanolamine and sodium chloride were purchased from SD fine chemicals, Mumbai, India; Dialysis membrane was procured from Sigma Aldrich, USA.

\section{Screening of components}

Table 1: Solubility of Cyclobenzaprine in various oils.

\begin{tabular}{|c|c|c|}
\hline S.No & Name of the oil & Solubility $(\mathbf{m g} / \mathbf{m l})$ \\
\hline 1 & Castor oil & $1.2 \pm 0.18$ \\
\hline 2 & Orange oil & $6.2 \pm 0.22$ \\
\hline 3 & Turpentine oil & $2.8 \pm 0.14$ \\
\hline 4 & Nutmeg oil & $2.5 \pm 0.12$ \\
\hline
\end{tabular}

The solubility of cyclobenzaprine in various oils (castor oil, orange oil, turpentine oil and nutmeg oil), surfactant (tween 80 ) and co-surfactants (span 80, polyethylene glycol, propylene glycol and ethanol) were determined by dissolving excess amount of cyclobenzaprine in $2 \mathrm{ml}$ of each of the selected oils, surfactants and co surfactants in $5 \mathrm{ml}$ capacity stoppered vials separately and mixed for 10 minutes using a vortex mixer. Combination of oils was also taken for determination of solubility. The mixture containing vials were then kept at $37{ }^{\circ} \mathrm{C}$ in an isothermal shaker (Nirmal International, Delhi, India) for 24 hours to attain equilibrium. The equilibrated samples were centrifuged at $3000 \mathrm{rpm}$ for 15 minutes. The supernatant was taken and filtered through a $0.45 \mu$ membrane filter and the concentration of cyclobenzaprine was determined by UV spectrophotometer at $290 \mathrm{~nm}$. The solubility study is shown in Table 1.

\section{Pseudo ternary phase diagram study}

On the basis of the solubility studies orange oil was selected as oil phase. Tween 80 and ethanol were selected as surfactant and co surfactant respectively. Distilled water was used as an aqueous phase. Surfactant and co surfactant (Smix) were mixed in different ratios $(1: 3 ; 1: 2 ; 1: 1 ; 2: 1 ; 3: 1$ and $4: 1)$ were used to optimize and determine the optimum ratio. Optimization of surfactant and co surfactant is shown in Table 2. For each phase diagram, oil and specific Smix ratio was mixed well in different weight ratios from 1:9 to 9:1 in different glass vials. Seventeen different combinations of oil and Smix , 1:9, 1:8, 1:7, 1:6, 1:5, 1:4, $1: 3,1: 2,1: 1,2: 1,3 ; 1,4: 1,5: 1,6: 1,7: 1,8: 1$ and $9: 1$ were made so that maximum ratios were covered for the study to delineate the boundaries of phases precisely formed in the phase diagrams. Pseudo ternary phase diagrams of oil, Smix and aqueous phase were developed using aqueous titration method under magnetic stirring [12]. Slow titration was done after each addition of aqueous phase to each ratio and observed visually for its transparency in terms of clarity. Optimization of surfactant: cosurfactant and oil: $\mathrm{S}_{\text {mix }}$ ratio is shown in (Table 2-4).

Table 2: Optimization of surfactant: co-surfactant.

\begin{tabular}{|c|c|c|}
\hline Surfactant & Cosurfactant & Observation \\
\hline Tween 80 & Span 80 & White \& cloudy \\
\hline Tween 80 & PEG 400 & Cloudy \\
\hline Tween 80 & Propylene glycol & Transparent with light yellow \\
\hline Tween 80 & Ethanol & Transparent \\
\hline
\end{tabular}

Table 3: Optimization of Surfactant: Co-surfactant $\left(\mathrm{S}_{\text {mix }}\right)$ Ratio

\begin{tabular}{|c|c|c|}
\hline Tween 80 & Ethanol & Physical state of nanoemulsion \\
\hline 1 & 0 & No emulsion formation \\
\hline 1 & 3 & Transparent (low stability) \\
\hline 1 & 2 & Slightly transparent \\
\hline 1 & 1 & Slightly transparent \\
\hline 2 & 1 & Transparent \\
\hline 3 & 1 & Slightly yellowish and transparent \\
\hline 4 & 1 & Yellowish and transparent \\
\hline
\end{tabular}

Table 4: Optimization of Oil: Smix Ratio

\begin{tabular}{|c|c|c|c|}
\hline $\begin{array}{c}\text { Code } \\
\text { No }\end{array}$ & $\begin{array}{c}\mathbf{S}_{\text {mix }} \\
\text { Ratio }\end{array}$ & Oil : $\mathbf{S}_{\text {mix }}$ ratio & Physical state of Nanoemulsion \\
\hline F1 & $2: 01$ & $9: 01$ & Milky N.E \\
\hline F2 & $2: 01$ & $8: 01$ & Milky N.E \\
\hline F3 & $2: 01$ & $7: 01$ & Milky N.E \\
\hline F4 & $2: 01$ & $6: 01$ & Milky N.E \\
\hline F5 & $2: 01$ & $5: 01$ & Milky N.E \\
\hline F6 & $2: 01$ & $4: 01$ & Milky N.E \\
\hline F7 & $2: 01$ & $3: 01$ & Milky N.E \\
\hline F8 & $2: 01$ & $2: 01$ & Pale bluish white \\
\hline F9 & $2: 01$ & $1: 01$ & Pale bluish white \\
\hline F10 & $2: 01$ & $1: 02$ & Translucent NE \\
\hline F11 & $2: 01$ & $1: 03$ & Translucent NE \\
\hline
\end{tabular}




\begin{tabular}{|l|l|l|l|}
\hline F12 & $2: 01$ & $1: 04$ & Transparent NE \\
\hline F13 & $2: 01$ & $1: 05$ & Transparent NE \\
\hline F14 & $2: 01$ & $1: 06$ & Transparent NE \\
\hline F15 & $2: 01$ & $1: 07$ & Transparent NE \\
\hline F16 & $2: 01$ & $1: 08$ & Transparent NE \\
\hline F17 & $2: 01$ & $1: 09$ & Transparent NE \\
\hline
\end{tabular}

\section{Drug-excipients compatibility studies}

Excipients are integral components of all dosage form. Compatibility between the drug and excipients are determined by DSC, FT-IR, TLC and UV techniques. Fourier Transform Infrared Spectroscopy (FTIR) and UV spectrophotometry studies were used for the determination of physicochemical compatibility and interactions between the drug and excipients. The earlier reports on drug-excipient interactions recommended that $1: 1$ ratio of drug and excipient maximizes the possibility of interaction and helps in easier detection of incompatibilities [13]. Therefore, in the present study 1:1 ratio was used for the preparation of physical mixtures and analyzed for compatibility studies.

\section{Preparation of cyclobenzaprine nanoemulsion}

Homogeneous organic phase was prepared by dissolving drug in the oil phase. Then the selected $S_{\text {mix }}$ was added to the oil phase and stirred in the magnetic stirrer for 5 minutes. The homogenous organic phase was slowly injected into the aqueous phase under magnetic stirring (4000rpm); the o/w emulsion was instantaneously formed by diffusion of the organic solvent in the external aqueous phase leads to formation of nanodroplets and to attain the equilibrium. Magnetic stirring was continued for 8 hours at room temperature for the complete evaporation of the water miscible solvent.

\section{Preparation of cyclobenzaprine nanoemulgel}

Cyclobenzaprine nanoemulgel was prepared by dispersing $1 \mathrm{~g}$ of the Carbopol-934 in sufficient quantity of distilled water, after complete dispersion of the polymer it was kept in dark for 24 hours for swelling. Then the cyclobenzaprine loaded nanoemulsion was slowly added to the viscous solution of Carbopol-934 under magnetic stirring. Other ingredients isopropyl alcohol and PEG-400 were added and mixed well; then the $\mathrm{pH}$ was adjusted to neutral with triethanolamine. The composition of cyclobenzaprine nanoemulgel is given in Table 5 .

Table 5: Composition of Cyclobenzaprine gel.

\begin{tabular}{|c|c|}
\hline $\begin{array}{c}\text { Cyclobenzaprine Hydrochloride Nanoemul } \\
\text { gel }\end{array}$ & $\begin{array}{c}\text { Ingredients } \\
(\mathbf{1 0 0 g})\end{array}$ \\
\hline Cyclobenzaprine hydrochloride nanoemulsion & 2 \\
\hline Carbopol 934 & 1 \\
\hline Isopropyl alcohol & 10 \\
\hline Polyethylene glycol & 10 \\
\hline Triethanolamine & 0.5 \\
\hline Distilled water & Q.S \\
\hline
\end{tabular}

\section{Characterization of cyclobenzaprine nanoemulgel}

Globule size distribution \& polydispersity index: The mean globule size of the nanoemulsion was determined by Photon Correlation Spectroscopy (PCS) using a Zeta sizer 3000 (Malvern Instruments, UK). This analytical results reveals the mean diameter of the particle at $25{ }^{\circ} \mathrm{C}$, and at an angle of 90 degree $(n=10)$. The PCS analysis yields a mean diameter (z-average) as a light intensity-weighed size of bulk population and the polydispersity index as a measurement for the width of a globule size distribution.

Zeta potential: The electrophoretic mobility was obtained by a Laser Doppler Anemometer connected with the Malvern zeta sizer instrument. A suitable amount of sample (50-100 $\mu \mathrm{L})$ was diluted with $5 \mathrm{~mL}$ of water $(0.45 \mu \mathrm{m})$ and injected in the electrophoretic cell of the instrument where a potential of $\pm 150 \mathrm{mV}$ was set. The zeta potential values were calculated by the instrument software using Smoluchosky equation.

\section{Morphology by transmission electron microscopic (TEM)}

TEM helps to visualize the inherent matrix of individual globule and its shape. A drop of the suitably diluted sample was placed on to a holey carbon coated copper grid and left for 10 minutes. Then grid was kept inverted and a drop of phosphotungstic acid (PTA) was applied to the grid for $10 \mathrm{~s}$. Excess of PTA was removed by absorbing on a filter paper and the grid was analyzed using the TECNAI-10 (PHILIPS) operated at $70-80 \mathrm{kV}$ at $17500 \mathrm{x}$ magnification.

pH: The pH of the formulation was measured in a pH meter (Sartorius, Germany).

Viscosity: The viscosity of the nanoemulgel was determined by a viscometer (Brookfield DV-E viscometer,) which was rotated for $10 \mathrm{~min}$ at 100 maximum rotations per minute with spindle 61 (Shivhare, 2009).

Drug content: The amount of drug present in the cyclobenzaprine nanoemulgel was determined by dissolving $5 \mathrm{~g}$ of nanoemulgel in $25 \mathrm{ml}$ of phosphate buffer solution at $\mathrm{pH} 7.4$ by sonication. After sonication the solution was filtered, the filtrate was diluted suitably and the absorbance was measured at $273 \mathrm{~nm}$ in UV-Visible spectrophotometer (Shimadzu, Japan, Model: 1700), finally the percentage of drug content was calculated.

Spreadability: Spreadability test was performed by measuring the spreading diameter of the nanoemulgel between two glass slides. Weighed about $0.5 \mathrm{~g}$ of sample and placed at the centre (within a circle) of the glass plate, over which a second glass plate was placed and pressed between the two slides and measured the spreadability of the gel in $\mathrm{cm}$ after 5 minutes.

\section{In-Vitro release}

In-vitro release studies were carried out by using dialysis membrane bag method. The dialysis membrane was conditioned by soaking in phosphate buffer 7.4 for 8 hours. Cyclobenzaprine 


\section{Novel Approaches in Drug Designing \& Development}

nanoemulgel of about $3 \mathrm{~mL}$ was taken in the dialysis membrane and immersed in $200 \mathrm{~mL}$ of phosphate buffer solution ( $\mathrm{pH} 7.4$ ). A sample of $5 \mathrm{~mL}$ was withdrawn from the dissolution setup at regular intervals for 8 hours and an equal volume of phosphate buffer ( $\mathrm{pH}$ 7.4) was replaced to maintain a sink condition. Samples were analyzed by using UV spectrophotometer at $290 \mathrm{~nm}$ and the amount of drug release was calculated and compared with the marketed oral dosage form.

\section{Result and Discussion}

Formulation in the form of Nanoemulsion has higher solubilization capacity, better thermodynamic stability, long self-life, rapid onset of action and large interfacial areas for the drug action leads to provide optimum targeted drug delivery and reduced inter subject bioavailability variation. The drug cyclobenzaprine is a skeletal muscle relaxant, its pharmacological property suggest that it can be a very good potential drug candidate for the transdermal drug delivery. Our present study is to formulate nanoemulgel for transdermal drug delivery of cyclobenzaprine. The materials used in the formulation are chosen based on its acceptability, nonirritant and nonsensitizing to the skin. Oils, surfactants and co-surfactants are having different physico-chemical properties, but in combination with each other modify the characteristics of the resultant self-emulsifying drug delivery systems. The selected oil phase has important role that the drug should be maintain in the solubilized form. Non-ionic surfactants with relative high HLB value are widely recommended to form a stable nanoemulsion formulation due to its high hydrophilicity which assists the immediate formation of $\mathrm{o} / \mathrm{w}$ droplets and rapid spreading of the formulation in aqueous media. Surfactant alone some time may not able to give clear and stable nanoemulsion. If the formulation is slight cloudy it may show phase separation after few days, it can be avoided by inclusion of hydrophilic co-surfactant. This cosurfactant produces clear and aqueous stable nanoemulsion of interest. Ethanol was selected as co-surfactant, which has HLB value of 4.2. Screening of the components for the solubility of the drug in the oil, surfactant and co-surfactant are having important role. Solubility study was performed in different oils, surfactant and co-surfactant. The solubility of the cyclobenzaprine was found to be highest in orange oil when compared to the other oils. The drug was completely soluble in tween 80 and highest solubility of the drug was observed in ethanol. Hence, orange oil, tween 80 and ethanol were selected as oil, surfactant and cosurfactant respectively for the phase study.

Constructing phase diagram is time consuming and care is required to be taken to ensure the observations are not made on meta stable systems. Concentration of compounds for the preparation of nanoemulsion can be easily determined from phase diagram. Safety is a major determining factor in choosing a surfactant, as a large amount of surfactants may cause skin irritation. Hence from the phase diagram study results, the oil: $S_{\text {mix }}$ ratio of 1:5, 1:6 and 1:7 were chosen for the further studies. The required quantity of the drug was incorporated in nanoemulsion and observed visually for its transparency and flow ability. Transparency of the nanoemulsion is shown in Figure 1.

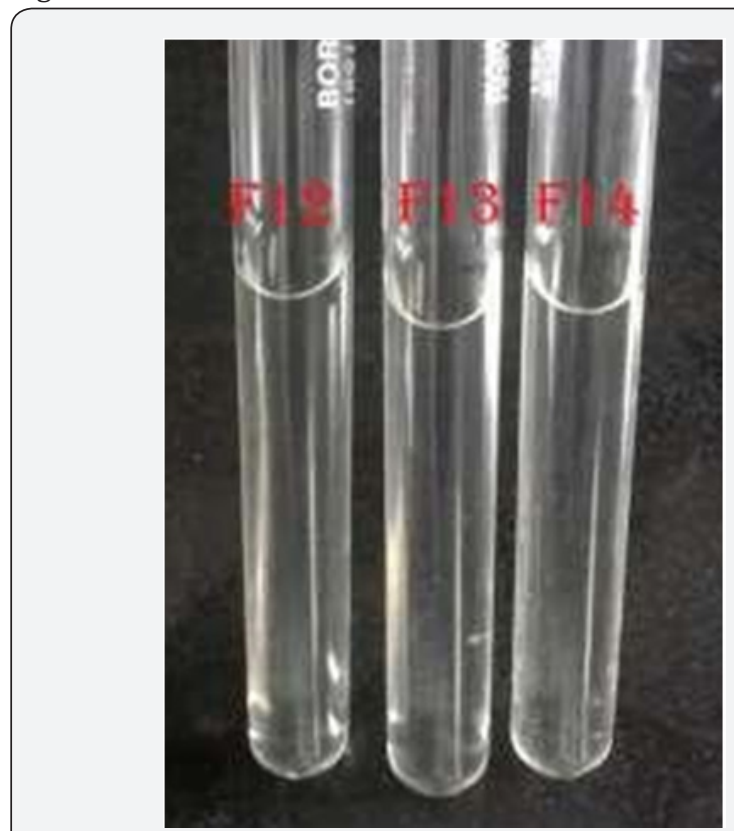

Figure 1: Transparency of Nanoemulsions formulation.

FT-IR and UV studies were performed to investigate chemical interactions between drug and the excipients. The FT-IR of cyclobenzaprine shows intense bands at 3000, 2980, 1500, 3100 and $860 \mathrm{~cm}^{-1}$ corresponding to the functional groups of alkenyl stretching, alkyl stretching, $\mathrm{NH}$ bending vibration of the tertiary amino group, aromatic $\mathrm{CH}$ stretching and bending vibrations respectively; these characteristic bands were not appeared in the formulation this might be due to the complete entrapment of the drug in the formulation excipients. No new bands or shift in characteristic peaks were appeared. In UV technique, the UV spectrum of drug is super impossible with the spectrum obtained with drug excipients mixtures and there is no change in the $\lambda_{\max }$ of $290 \mathrm{~nm}$ between the drug and drug excipients mixtures. FTIR and UV results revealed that there is no chemical interaction between the drug and the excipients used in the formulation and found to be compatible with each other (Data not shown).

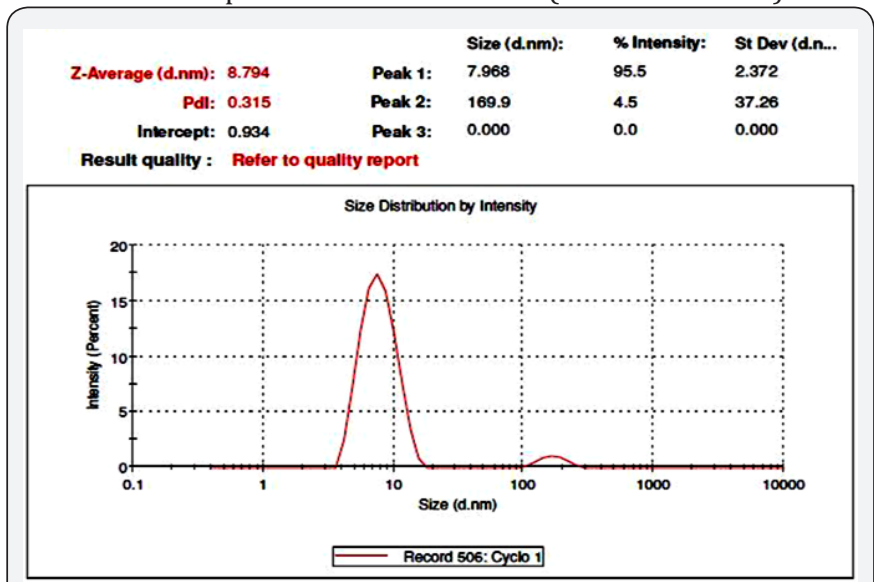

Figure 2: Globule size spectra of Nanoemulsion. 


\section{Novel Approaches in Drug Designing \& Development}

The polydispersity (PDI) of optimized formulations F12, F13 and F14 were measured and the value was found to be $0.315,0.177$ and 0.175 respectively; which indicates the broad distribution of globules and its homogencity. Globule size in the formulation either increases or decreases which can affect the drug release from the formulation and consequently its bioavailability. For emulsion based products, the globule size of droplets of the internal phase have an impact on the stability of emulsion itself. The mean globule size was found to be $38.79 \mathrm{~nm}$, $24.55 \mathrm{~nm}$ and $8.79 \mathrm{~nm}$ for formulation F12, F13 and F14 respectively. The globule size distribution for the formulation F14 is shown in Figure 2.

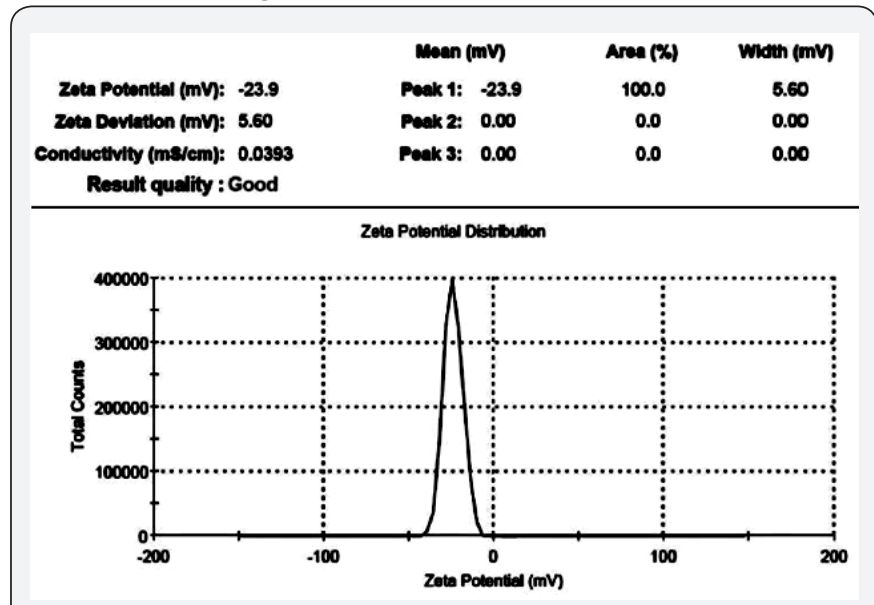

Figure 3: Zeta potential spectra of Nanoemulsion.

The zetapotential was determined by using Malvern zetasizer, it was found to be $-23.9 \mathrm{mV},-20.4 \mathrm{mV}$ and $-18.45 \mathrm{mV}$ respectively for formulation F12, F13 and F14 respectively. Nanoemulsion formulation consists of non-ionic surfactants which show relatively neutral charge, it means it will not affected by body membrane charge during absorption. The formulation shows an accepted value for good stability, the zeta potential distribution is shown in Figure 3.

TEM for the prepared formulations was examined using TECNAI-10 (PHILIPS) operated at $70-80 \mathrm{kV}$ at $17500 \mathrm{x}$ magnification. The TEM results showed that the globules were of nanometer in size range with uniform, spherical and smooth surface. The TEM image of the formulation F14 is shown in Figure 4. The $\mathrm{pH}$ values of the prepared formulation were found to be between 6.13 and 6.55 nearing neutral. The result reveals that the $\mathrm{pH}$ of the prepared Nanoemulsions is within the acceptable limits for topical application.

The viscosity of the formulation was found to be between $38.28 \mathrm{cps}$ and $45.25 \mathrm{cps}$. The viscosity was decreased when there is an increase in concentration of oil. The observed results are matching with the earlier reports [12]. Drug content of all formulations was in the range between 97.54 and $101.25 \% \mathrm{w} / \mathrm{w}$. Spreadability is an important parameter for the gel it should comes out from the tube. The spreadability of nanoemulgel formulation was determined; which exhibit the good spreadability when it applied on the inflamed skin with maximum slip and drag. The spreadability of the formulation was found to be $4.6 \pm 0.18 \mathrm{gcmS}^{-1}$.
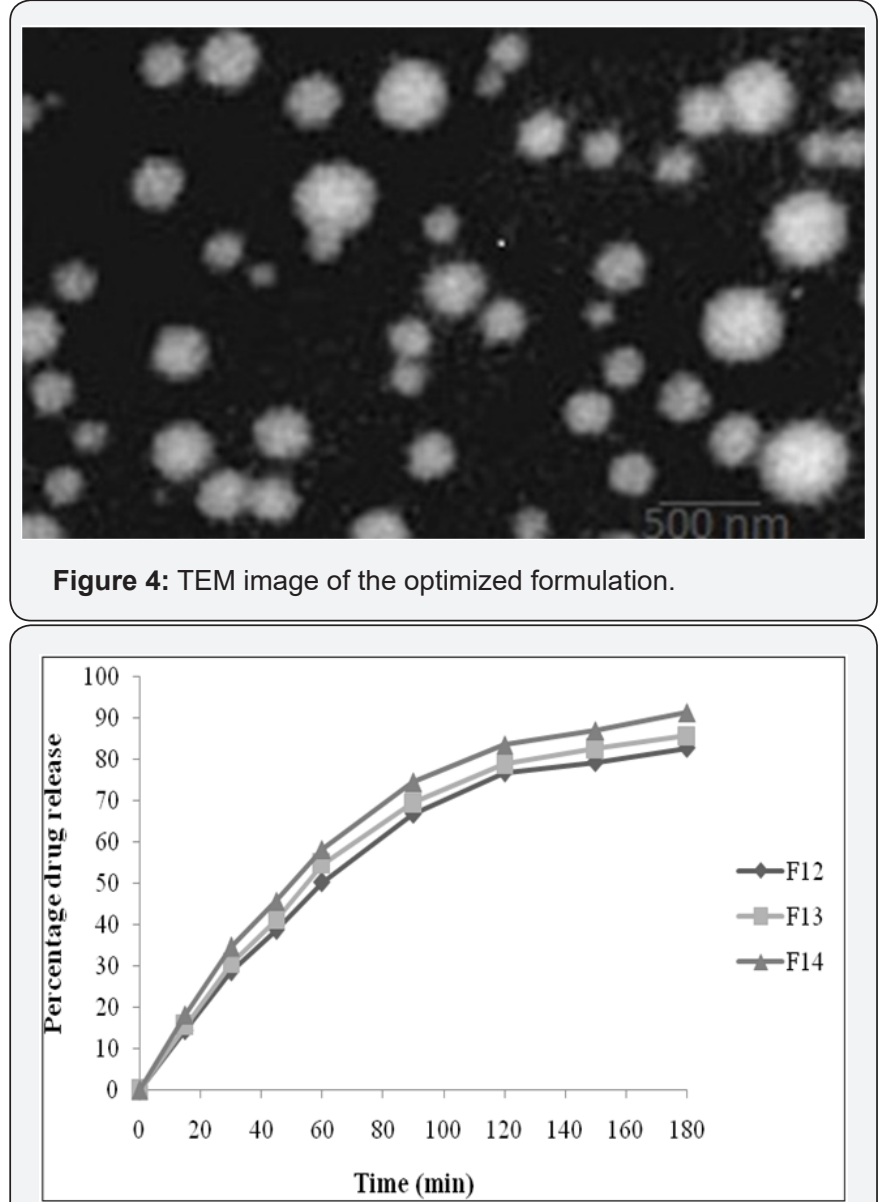

Figure 5: Comparative in-vitro release studies of the formulation F12, F13 and F14.

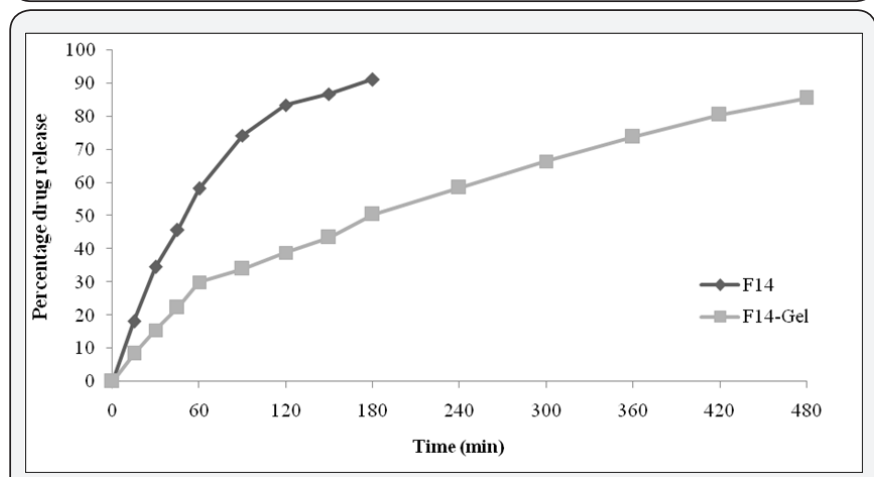

Figure 6: Comparative in-vitro release profile of the optimized Nanoemulsion (F14) and nanoemul gel.

The in-vitro release profile of cyclobenzaprine nanoemulsion is given in Figure 5. A burst release followed by a steady release has been observed and the drug release process ranked in the order of $\mathrm{F} 14>\mathrm{F} 13>\mathrm{F} 12$. The amount of drug released after 180 min were found to be $91.22 \%, 85.66$ and $82.63 \%$ respectively for F14, F13 and F12. Thus significant $(\mathrm{P}<0.5$, t-test $)$ higher drug release was observed for formulation F14 compared to others. The optimized formulation F14 followed the higuchi release kinetics that is based on diffusion controlled. According 
to the "n" value 0.158 obtained from korsemeyer peppas model, F14 followed non-fickian case II diffusion mechanism. The invitro release profile of cyclobenzaprine from its nanoemulgel formulation is compared with its nanoemulsion. The comparative in-vitro release is shown in Figure 6.

\section{Conclusion}

Cyclobenzaprine is drug used as skeletal muscle relaxant. Recently formulation in the form of nanoemulsion has attention in the drug delivery system due to its nanosize droplets with good stability. A suitable combination of the oil, surfactant and co-surfactant are the major consideration in the formulation of Nanoemulsion as transdermal drug delivery system. In this work, cyclobenzaprine nanoemulsion has been developed. The optimized nanoemulsion formulation, which exhibited maximum drug release, was formulated as novel nanoemulgel of cyclobenzaprine with suitable viscosity for topical administration. The in-vitro results revealed that the developed nanoemulsion based gel containing cyclobenzaprine hydrochloride has great potential to provide better therapeutic effect locally through topical application.

\section{References}

1. Tharwat T, Izquierdo P, Esquena J, Solans C (2004) Formulation and Stability of Nanoemulsions. Adv Colloid Interface Sc 108-109: 303-318.

2. Debnath S, Satayanarayana, Vijay Kumar G (2011) Nanoemulsion-A Method to improve the solubility of lipophilic drugs. Int J Advances in Pharmaceutical Sci 2: 2-3.

3. Shah P, Bhalodia D, Sheti P (2010) Nanoemulsion: A Pharmaceutical Review. Sys Rev Pharm 1(1): 24-32.
4. Doh HJ, Jung Y, Balakrishnan P, Cho HJ, Kim DD (2013) A novel lipid nanoemulsion system for improved permeation of granisetron. Colloids Surf B Biointerfaces 101: 475-480.

5. Chouksey RK, Pandey HK, Maithil A, Jain AK (2011) Nanoemulsion: A Review. Inventi Rapid: Pharm Tech 2(1): 1-23.

6. Guilatt LR, Lambert G (2005) Cationic emulsion technology for lipophilic drug delivery. Drug Delivery report Autumn winter 71-72.

7. Lawrence MJ, Reece GD (2000) Microemulsion-based media as novel drug delivery systems. Adv Drug Deliv Rev 45(1): 89-121.

8. Eccleston J (1994) Microemulsions. In: Swarbrick J and Boylan JC (Eds.), Encyclopedia of Pharmaceutical Technology. Marcel Dekker, New York, UK, 9: 375-421.

9. Kawakami K, Yoshikawa T, Moroto Y, Kanaoka E, Takahashi K, et al. (2002) Microemulsion formulation for enhanced absorption of poorly soluble drugs II. J Control Release 81(1-2): 75-82.

10. Constantinides PP (1995) Lipid microemulsions for improving drug dissolution and oral absorption and biopharmaceutical aspects. Pharm Res 12(11): 1561-1572.

11. Chouksey R, Jain AK, Pandey H, Maithil A (2011) Development and bioavailability studies of atorvastatin Nanoemulsion. Int J of Pharm \& Life Sci (IJPLS) 2(8): 982-988.

12. Baboota S, Shakeel F, Ahuja A, Ali J, Shafiq S (2007) Design development and evaluation of novel nanoemulsion formulations for transdermal potential of celecoxib. Acta Pharm 57(3): 315-332.

13. Lakshmana Prabu S, Shnanawaz S, Dinesh Kumar C (2008) Compatibility Studies Between Duloxetine Hydrochloride and Tablet Excipients Using Thermal and Non-thermal Methods. J Pharm Res 7: 20-23.

\begin{tabular}{l} 
Your next submission with Juniper Publishers \\
will reach you the below assets \\
- Quality Editorial service \\
- Swift Peer Review \\
- Reprints availability \\
- E-prints Service \\
- Manuscript Podcast for convenient understanding \\
- Global attainment for your research \\
- Manuscript accessibility in different formats \\
( Pdf, E-pub, Full Text, Audio) \\
- Unceasing customer service \\
Track the below URL for one-step submission \\
https://juniperpublishers.com/online-submission.php \\
\hline
\end{tabular}

